\title{
PT-axisymmetry for extraordinary field confinement
}

\author{
M. Botey*a, Waqas. W. Ahmed ${ }^{\mathrm{a}}$, R. Herrero ${ }^{\mathrm{a}}$, K. Staliunas ${ }^{\mathrm{a}, \mathrm{b}}$ \\ a Departament de Física i Enginyeria Nuclear, Universitat Politècnica de Catalunya, Catalonia \\ b'Institució Catalana de Recerca i Estudis Avançats, Catalonia
}

\begin{abstract}
Optical Parity-Time (PT-) symmetric systems support unusual properties when the symmetric coupling between internal modes is broken. We propose a new class of PT-axisymmetric systems which lead to a simultaneous extraordinary field enhancement and localization at the symmetry center. The effect is based on the asymmetric radial coupling of outward to inward propagating waves of the complex structure. We explore such optical potentials in 1D and 2D combining gain/loss and index modulations, which could have actual realizations in nanophotonic structures. As a direct application, we show how to render a broad aperture vertical-cavity surface-emitting lasers (VCSEL) into a bright and narrow beam source.
\end{abstract}

Keywords: PT-symmetry, light confinement, VCSEL, nanostructures.

\section{INTRODUCTION}

Dispersion and diffraction of light beams, a phenomenon limiting the performance of many linear and nonlinear optical devices. Artificial structured materials presenting a spatial modulation of the refraction index and the gain or loss on the wavelength scale have demonstrated the ability to tailor the dispersion, being ideal candidates to overcome this drawback [1-6]. However, in real materials gain-loss and refractive index are not independent due to the non-instantaneous and causal response of the medium, as described by the Kramers-Kroning relation. For instance, introducing a weak modulation on a given actual material results in either in-phase or anti-phase gain and index modulations, depending on frequency. PTsymmetric materials are complex materials in which the index and gain-loss modulations are dephased a quarter of the spatial period of the modulation. First introduced as a curiosity in quantum mechanics [7], PT-symmetric systems are attracting interest especially in optics, for holding new intriguing properties and supporting novel unusual effects, specifically when the PT-symmetry is broken. The PT-symmetry condition requires that the complex refractive index, representing the actual refractive index and the gain-loss distribution, fulfils the condition: $n(r)=n *(-r)$. Indeed, the wellknown Photonic Crystals (PhCs), with purely real modulations of the refractive index satisfy the PT-symmetry conditions. On the other extreme, systems with a constant real refractive index, holding only Gain-Loss Modulation (GLM) may also be regarded as a particular class of PT-symmetric system. However, in both cases, the system behavior is perfectly symmetric. The most interesting situations arise when both the real refractive index and the gain-loss are modulated. Especially when both modulations are balanced, the symmetric coupling between internal modes is broken. Then, the system becomes strongly unidirectional, leading to new features on light waves such as asymmetric unidirectional reflectors, invisible materials or input-dependent chirality in 2D systems [8-14]. For the simplest harmonic modulation, an optical PT-symmetric system may be describes by a complex potential such as: $n(x)=n_{\operatorname{Re}} \cos (q x)+i n_{\text {Im }} \sin (q x)$. At the PT-symmetry breaking point, when the amplitudes of both modulations are equal, $n_{\mathrm{Re}}=n_{\mathrm{Im}}$, the complex refractive index may be expressed as: $n(x)=n \exp ( \pm \mathrm{i} q x)$. In this case, the resonant propagation modes, exp (ikx) and exp (-ikx), with $q=2 k$, are unidirectionally coupled, leading to the new asymmetric properties.

We propose a new class of PT-symmetric optical potentials with axisymmetry, for the concentration and enhancement of the optical field. The basic principle can be seen just considering a transversal cut of the structure, or equivalent a simple 1D situation as the one represented on Fig.1a. On the $x$-axis the system is divided into two half-spaces: $x<0$ and $x>0$ with symmetric complex refractive index distribution. Such a configuration yields to a unidirectional coupling with opposite directions in the two half-spaces, being the coupling "to the left" on the right half-plane, and "to the right" on the left half-plane. Therefore, an accumulation of the field can be intuitively expected at the symmetry center, $x=0$. The same idea applies to 2D (see Fig.1b) if the radial coupling between incoming and outgoing waves is asymmetric. We analyze spatial effects on beam propagation in such complex structures, both in 1D and 2D. We note that the physical realizations of the two above discussed arrangements in 1D and 2D are nowadays available in microphotonics, by special microfabrication techniques of microchip- and vertical-cavity surface-emitting lasers. 


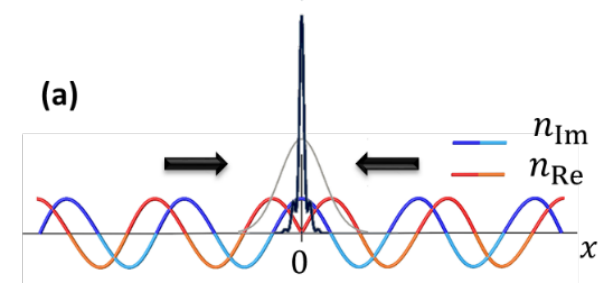

(b)

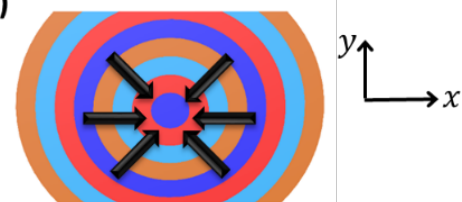

Figure 1. Local unidirectional coupling: (a) Transversal 1D cut of the axisymmetric complex optical potential, where $n_{\mathrm{Re}}$ is the real index modulation, and $n_{\text {Im }}$ is the gain-loss modulation. As schematically represented, a field concentration may be expected at $x=0$ with such a harmonic modulation or alternatively combining four different materials. (b) 2D analogous axisymmetric configuration, where the arrows indicate the inwards coupling.

\section{PT-AXISYMMETRIC POTENTIAL}

As the simplest PT-axisymmetric potential we may consider a harmonic optical refractive index satisfying the asymmetric inwards coupling, expressed as:

$$
n(r)=n_{R e} \cos (r)-i n_{I m} \sin (r)
$$

where, for simplicity, we use normalized spatial amplitudes for the real and imaginary components of the complex refractive index; $n_{\mathrm{Re}}$ and $n_{\mathrm{Im}}$. The ratio between both amplitudes is expected to confer the asymmetric character to the system.

We shall study the propagation of a light beam in such structure by considering the general paraxial electromagnetic field equation as a mathematical model, including diffraction and the potential, as:

$$
\partial_{t} A(r, t)=\mathrm{i} \nabla_{\perp}^{2} A(r, t)+\mathrm{i} n(r) A(r, t)
$$

where $A(r, t)$ is the normalized slowly varying amplitude envelope of the complex electromagnetic field. The two important parameters determining the spatial and temporal dynamics of such systems described by eq. 2 , are $n_{\mathrm{Re}}$, and $n_{\mathrm{Im}}$.

\section{1D ANALYSIS}

Before fully analyzing the PT-axisymmetric case we shall start considering a 1D case, that is to say a potential in the form: $n(x)=n_{R e} \cos (|x|)-i n_{I m} \sin (|x|)$ in eq.(2). We numerically integrate the paraxial wave equation using the split step method, for a Gaussian initial beam in the form: $A(x)=\exp \left(-(x / w)^{2}\right)$ where $w$ is the width of the initial beam profile. We observe that such a system may develop an extreme localization at $x=0$, exhibiting an exponential temporal growth due to the linear nature of system. We initially explore the parameter space $\left(n_{\text {Re }}, n_{\text {Im }}\right)$. The envelope of the final field, after a sufficient propagation time, may be expressed as: $A(x) \approx \mathrm{e}^{\mathrm{i} \omega t} \mathrm{e}^{\mathrm{i} k 6 x 6}$ where $k=k_{R e}+\mathrm{i} k_{I m}$ and $\omega=\omega_{R e}+\mathrm{i} \omega_{I m}$ are both complex numbers. Note that while $k_{R e}$ is the spatial dominant mode $k_{I m}$ denotes the spatial localization exponent. Analogously, respectively, $\omega_{\mathrm{I} m}$ and $\omega_{R e}$ stand for the temporal growth exponent and-temporal oscillation frequency, respectively. Therefore, we obtain the localization exponent (which we shall refer as localization) after a sufficient long time evolution, $t$, as: $k_{I m}=\frac{d}{d x}[\log (A(x, t)]$. The temporal exponential growth, at $x=0$ (referred as growth throughout the text) may also be determined as: $\omega_{I m}=\frac{d}{d t}[\log (A(0, t)]$. Such two main quantities fully characterize the field dynamics in the complex system, and are therefore used to asses and identify the field localization and enhancement regimes in the parameter space $\left(n_{\text {Re }}, n_{\text {Im }}\right)$. The results for $1 \mathrm{D}$ case are presented on Fig. 2.

Figures $2 \mathrm{a}$ and $2 \mathrm{~b}$ provide the localization and growth maps, respectively. We observe that the largest growth occurs at the top right corner, coinciding with the simultaneous field enhancement on the same area of the parameter space, i.e. on the top right corner of both maps. To illustrate the effect of the PT-potential on the spatio-temporal field dynamics, we consider three representative points, within and outside the field localization regime, (c), (d) and (e), sharing the same amplitudes 
of the gain/loss and index modulations, but with different signs. While the potential leads to an inward coupling of the wavevectors in point (c), the situation is reversed in points (d) and (e) where the coupling is outwards. The spatial field profile at (c) evidences a sharp localization of the field with an enhancement factor of $10^{22}$ at the center. On the contrary, while at (d) the field is spread. The field representation in logarithmic scale shows the expected exponential field localization in (c) and a flat field distributions for the other two cases. Also the temporal evolution of field is exponentially growing in (c), but becomes flat after a short transient in (d) or (e). All the spatial field spectra, as calculated from the Fourier transform, are also provided, showing integer dominant modes for (d) and (e). In (c), due to the strong exponential decay, the Fourier transform does not provide the half-integer dominant modes; for a deeper analysis using the Laplace transform, see Ref. [18].
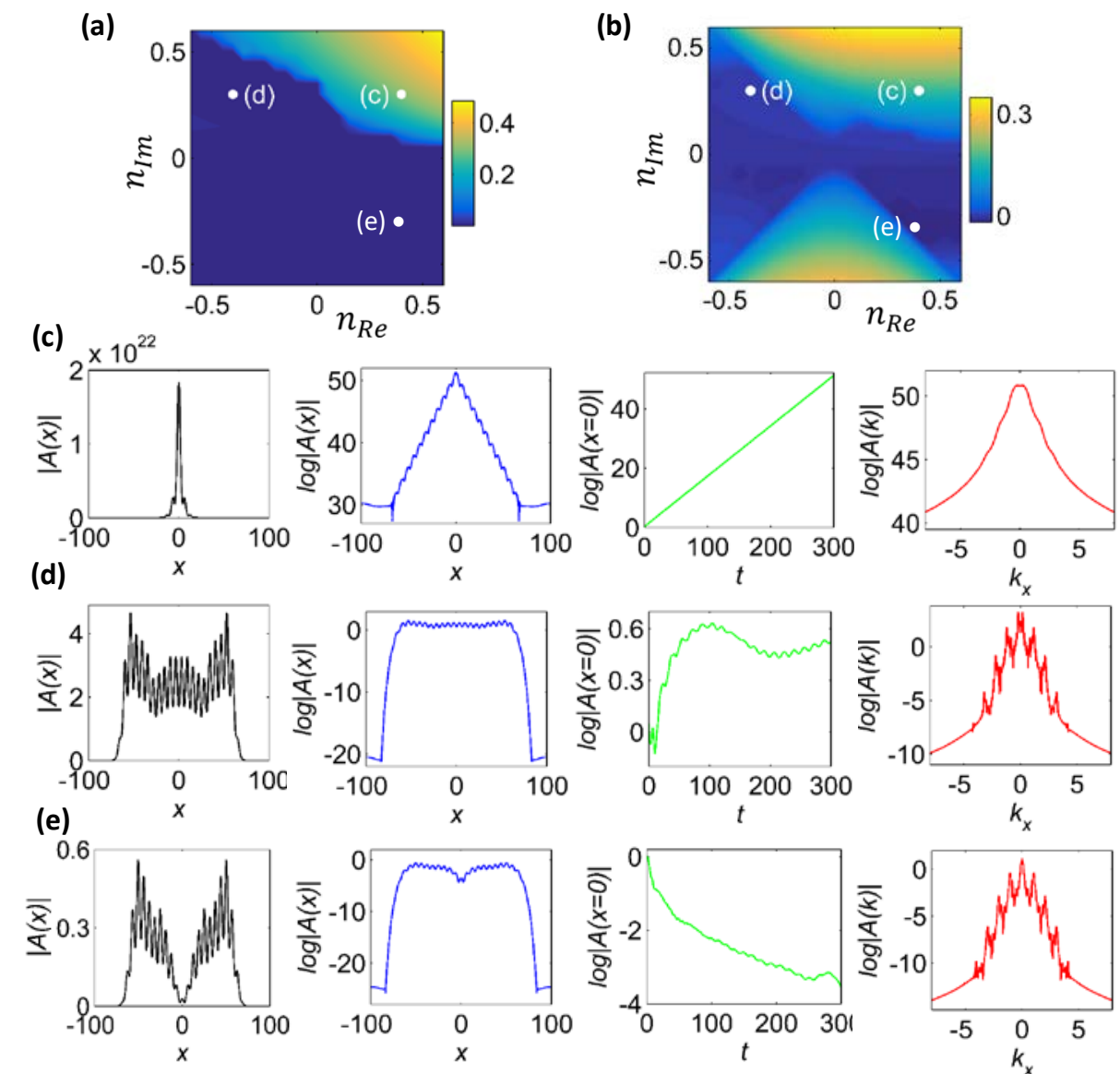

Figure 2. 1D field concentration (a) Localization $\left(k_{\operatorname{Im}}\right)$ map, calculated after a sufficient long time ( $\mathrm{t} \sim 300$ units) in the parameter space $\left(n_{\mathrm{Re}}, n_{\mathrm{Im}}\right)$. (b) Growth map $\left(\omega_{\mathrm{Im}}\right)$ at the center $(x=0)$, in the parameter space $\left(n_{\mathrm{Re}}, n_{\mathrm{Im}}\right)$. Three representative points marked as (c),(d),(e) correspond to the parameter sets $(0.4,0.3),(-0.4,0.3)$, and $(0.4,-0.3)$, respectively. In all cases, the spatial field profiles in linear and logarithmic scale are shown on the first and second columns of (c),(d),(e) while the third and forth columns represent the temporal evolution of the field at the center and the corresponding spatial field spectrum.

\section{FIELD LOCALIZATION BY 2D PT-AXISYMMETRY}

The analysis of the 2D PT-axisymmetric optical system provides very similar results, see Fig.3. We observe a strong field enhancement at $r=0$, which may be clearly attributed to the asymmetric radial coupling between inward and outward propagation waves. We note that the proposed 2D PT-axisymmetric system represented in Fig.1b is equivalent to a coaxial configuration, see the upper row of Fig.3. While on the first case the structure leads to a field concentration and enhancement at $r=0$ due to the radial coupling of fields evolving in time, the field concentration on the second case occurs in propagation along the z-axis. The axial cross-sectional profile of the field, in logarithmic scale is not linear, due 
to a factor $1 / r$ of the axial symmetry. The parameter space is explored and the map of the linearized localization exponent of the axial cross-sectional profile, obtained from $A(r) \sqrt{r}$, is shown in Fig. 3a. A high growth and extreme localization regime is found simultaneously on the top right quadrant of Fig. 3a and 3b, for $n_{\mathrm{Re}}>0 n_{\mathrm{Im}}>0$, approximately corresponding to the $1 \mathrm{D}$ results. These maps indicate that the overall scenario remains analogous to $1 \mathrm{D}$. The 2D field profiles in linear and logarithmic scales, and the spectral profile, are determined for the parameter set of point (c) in Fig. 3a, within the localization regime, and depicted in Fig. 3c(i-iii) as an example of strong field confinement around $r=0$. The general conclusion is that the situation in this $2 \mathrm{D}$ case is analogous to $1 \mathrm{D}$ with the minor advantage of larger growth exponents but with slightly smaller localization.

Note that while the results are analogous to the simple 1D case, this 2D geometry is more realistic as it allows thinking in possible implementations in actual systems. In fact, as a first direct application, we expect the proposed effect be useful to improve the beam quality of emission from broad aperture lasers.

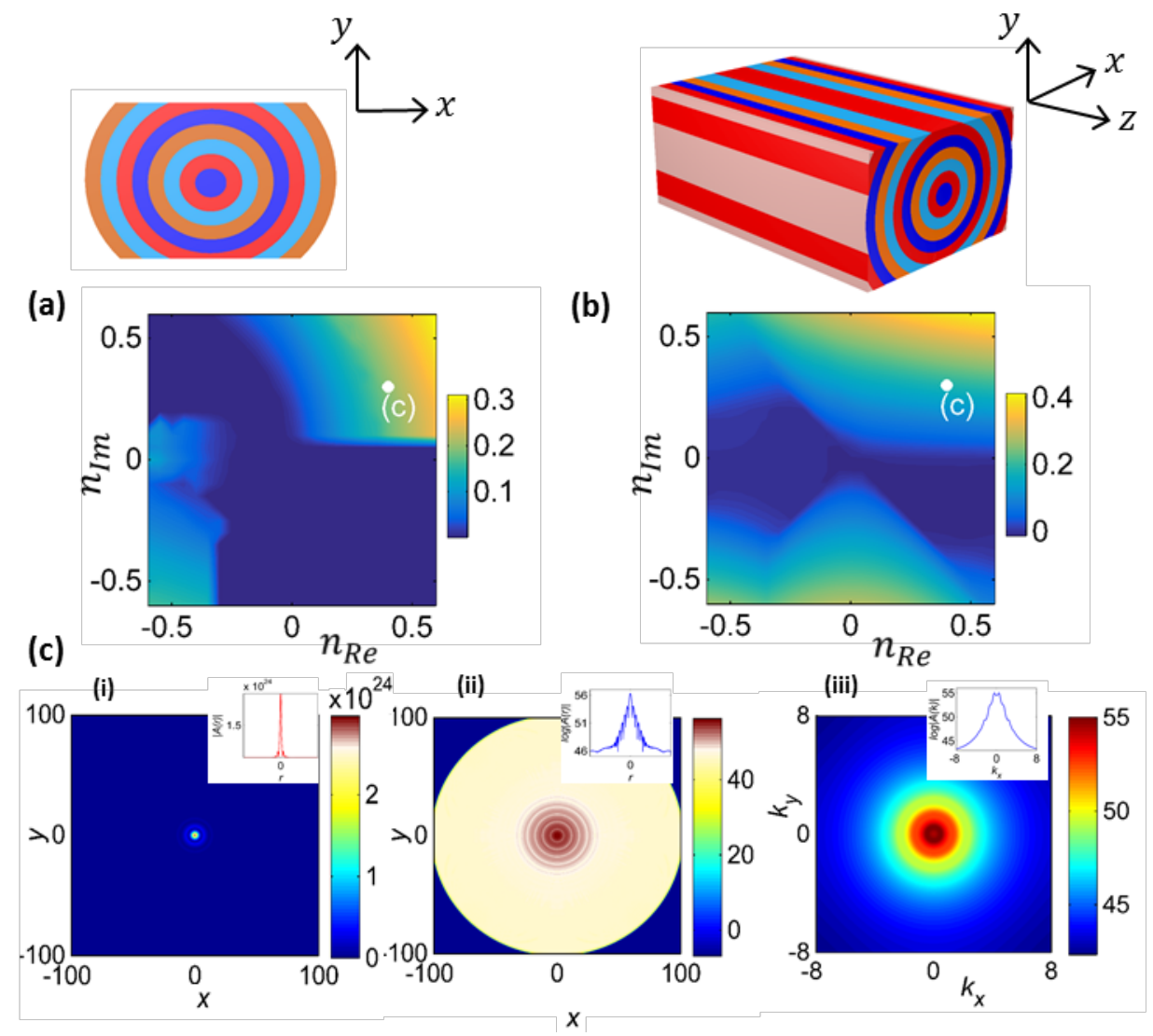

Figure 3. (a) 2D PT-axisymmetric structure, with asymmetric spatial coupling of the fields evolving in time (d) analogous 2D coaxial index and gain/loss modulations, for fields propagating along $z$. The arrows in (a) and (b) indicate the direction of the asymmetric mode coupling. (b) Localization map of the axial cross-section profile of the field $A(r) \sqrt{r}$ for 2D, calculated after sufficient long time ( $t \sim 300$ units) in parameter space $\left(n_{\mathrm{Re}}, n_{\mathrm{Im}}\right)$. (c) Growth map of at the center $(r=0)$ in parameter space. (d) 2D spatial field profiles in (i) linear and (ii) logarithmic scales and (iii) far field in logarithmic scale. The insets show the corresponding axial cross-sectional profiles.

\section{PT-AXISYMMETRC BRAOD APPERTURE MICROLASER}

Broad aperture microlasers, and VCSELs among them, are relevant laser sources however suffering from a major drawback a poor beam quality due to the lack of an intrinsic transverse mode selection mechanism. In the simplest approximation, the field dynamics of VCSELs with the proposed complex potential profile, $U(r)$, satisfying the particular symmetry 2D axisymmetric conditions, can be described by complex Ginzburg-Landau equation [17]: 


$$
\partial_{t} A(r, t)=\left(p-|A|^{2}\right) A+i \nabla_{\perp}^{2} A(r, t)+i U(r) A(r, t)
$$

where $A(r, t)$ is the envelope of complex field distributed in space, $r$, and $p$ is pump parameter. The nonlinearity is due to gain saturation. The complex potential profile, $U(r)$, satisfies the local PT-symmetry and global P-symmetry conditions for 1D and 2D axisymmetric systems as discussed above. Such nonlinear systems generally shows saturation with uniform field distribution for $p>0$. However, the field localization and enhancement in such situation is possible at the center for $p<0$ by applying a PT-axisymmetric potential which could be realized with an active gain-layer and/or a profiled (microcorrugated) mirror with asymmetric spatial coupling of the fields evolving in time, see Fig.4b. The results for a particular parameter set are provided in Fig. 4. The spatial field profile showing the concentration at the center, $r=0$, reveals that this 2D PT-axisymmetry localizes the field, the linear logarithmic slope an exponential growth, see Fig.4c, and the phase of the field increasing with jumps from $-\pi$ to $+\pi$ - clearly showing the inwards propagation, see Fig.4d.

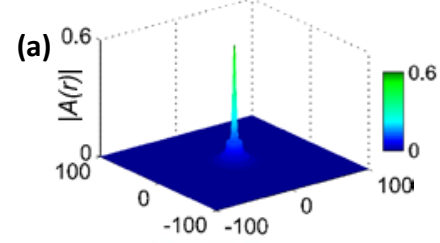

(b)

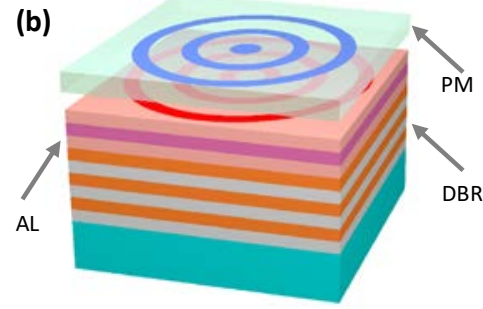

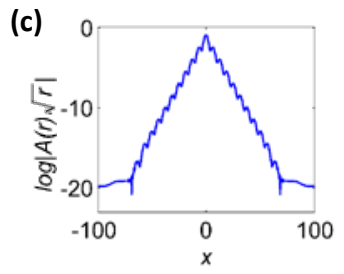

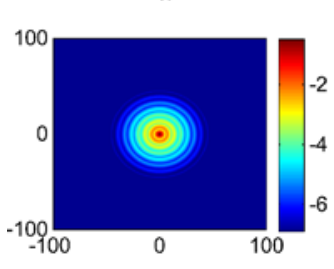

(d)
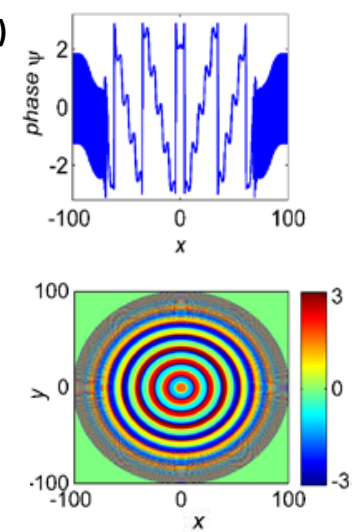

Figure 4. 2D PT- axisymmetric VCSEL (a) 3D visualization of the VCSEL output field profile calculated after the steady state ( $\mathrm{t} \sim 150$ units) for $p=-0.1$ and parameter set $(0.4,0.4)$ concentrated and enhanced at $r=0$ (b) Schematic representation of a modified PT-axisymmetric VCSEL; PM-Profiled Mirror, DBR-Distributed Bragg Reflector, AL-active layer. (c) 1D and 2D field profiles in logarithmic scale. (d) 1D and 2D distributions of the phase.

\section{CONCLUSIONS}

We propose a novel class of $P T$-axisymmetric systems that provide a versatile platform for strong field localization and enhancement around the double symmetry point. Such systems have the capability to simultaneously localize and enhance the field around a point of merging of the two symmetries due to the asymmetric coupling of inward and outward waves. This light-matter interaction phenomenon may find remarkable applications in many linear and nonlinear devices where a high degree of localization is essentially desirable such as: beam focusing, narrow beam enhancement, laser emission, switching in nanostructures etc.

One of the most promising applications of the idea is to use the PT-localization property to regularize the fields in broad aperture lasers, in particular in VCSELs. Broad aperture lasers, especially the semiconductor lasers, are known to result in spatial pattern formation $[15,16]$, which is usually an undesirable effect from the technological viewpoint. Different techniques to regularize the spatial structure of the emitted field are being studied, in particular the spatial modulation of the amplifying media $[17,18]$. The PT-like modulation proposed here could serve as advanced technique to regularize the spatial structure of output radiation.

\section{ACKNOWLEDGEMENTS}

We acknowledge financial support by Spanish Ministerio de Ciencia e Innovación, through project FIS2015-65998-C21-P; NATO SPS-985048 grant; and European Union FEDER Erasmus Mundus Doctorate Program Europhotonics (Grant No. 159224-1-2009- 1-FR-ERA MUNDUS-EMJD) supported the work of W.W.A. 


\section{REFERENCES}

[1] Staliunas, K., Herrero, R., and Vilaseca, R.,"Subdiffraction and spatial filtering due to periodic spatial modulation of the gain-loss profile," Phys. Rev. A 80, 013821 (2009).

[2] Botey, M., Herrero, R., and Staliunas, K., "Light in materials with periodic gain-loss modulation on a wavelength scale," Phys. Rev. A 82, 013828 (2010).

[3] Herrero, R., Botey, M., Radziunas, M., and Staliunas K., "Beam shaping in spatially modulated broad-area semiconductor amplifiers," Opt. Lett. 37 5253-5255 (2012).

[4] Radziunas, M., Botey, M., Herrero, R., and Staliunas, K., "Intrinsic beam shaping mechanism in spatially modulated broad area semiconductor amplifiers," Appl. Phys. Lett. 103, 132101 (2013).

[5] Kumar, N., Herrero, R., Botey, M., and Staliunas, K., "Flat lensing by periodic loss-modulated materials," JOSA B, 30(10), 2684-2688 (2013).

[6] Kumar, S., Herrero, R., Botey, M., and Staliunas, K., "Suppression of modulation instability in broad area semiconductor amplifiers, " Opt. Lett. 39(19), 5598-5601 (2014).

[7] Bender, C. M., and Boettcher, S., "Real spectra in non-Hermitian Hamiltonian having PT symmetry," Phys. Rev. Lett. 80, 5243-5246 (1998).

[8] Guo, A., Salamo, G. J., Duchesne, D., Morandotti, R., M., Aimez, V., Siviloglou, G. A., and Christodoulides, D. N., "Observation of PT-symmetry breaking in complex optical potentials," Phys. Rev. Lett. 103, 093902 (2009).

[9] Longhi, S., "Invisibility in PT-symmetric complex crystals," J. Phys. A, Math. Theor. 44, 485302 ( 2011 ).

[10] Ruter, C. E., Makris, K. G., El-Ganainy, R., Christodoulides, D. N., Segev, M., and Kip, D.,"Observation of parity-time symmetry in optics," Nat. Phys. 6, 192-195 (2010).

[11] Feng, L., Xu, Y.-L., Fegadolli, W. S., Lu, M.-H., Oliveira, J. E., Almeida, V. R., Chen, Y.-F., and Scherer, A., "Experimental demonstration of a unidirectional reflectionless parity-time metamaterial at optical frequencies," Nat. Mater. 12, 108-113 ( 2012).

[12] Lin, Z., Ramezani H., Eichelkraut, T., Kottos T., Cao, H., and Christodoulides, D. N.,"Unidirectional invisibility induced by PT-symmetric periodic structures," Phys. Rev. Lett. 106, 213901 (2011).

[13] Regensburger, A., Bersch, C., Miri, M-A., Onishchukov, G., Christodoulides, D. N., and Peschel, U., "Parity-time synthetic photonic lattices," Nature 488, 167-171 (2012).

[14] Turduev, M., Botey, M., Giden, I., Herrero, R., Kurt, H., Ozbay, E., and Staliunas, K., "Two-dimensional complex parity-time-symmetric photonic structures, “ Phy. Rev. A 91(2), 023825. (2015).

[15] Staliunas, K., and Sanchez-Morcillo V.J., " Transverse Patterns in Nonlinear Optical Resonators" Springer Verlag, Springer Tracts in Modern Physics 183, (2003).

[16] Tlidi, M., Staliunas, K., Panajotov, K., Vladimirov A. G., and Clerc, M. G., "Localized structures in dissipative media: from optics to plant ecology, " Phil. Trans. R. Soc. A 372, 20140101 (2014).

[17] Ahmed, W. W., Kumar, S., Herrero, R., Botey, M., Radziunas, M., and Staliunas, K., "Stabilization of flat-mirror vertical-external-cavity surface-emitting lasers by spatiotemporal modulation of the pump profile", Phys. Rev. A 92, 043829 (2015).

[18] Ahmed, W. W., Herrero, R., Botey, M., and Staliunas, K., "Locally parity-time-symmetric and globally paritysymmetric systems ," Phys. Rev. A 94, 053819 (2016). 